\title{
Effect of different trunk postures on scapular muscle activities and kinematics during shoulder external rotation
}

\section{$\operatorname{AUTHOR(S):~}$}

Miyakoshi, Kosuke; Umehara, Jun; Komamura, Tomohito; Ueda, Yasuyuki; Tamezawa, Toru; Kitamura, Gakuto; Ichihashi, Noriaki

\section{CITATION:}

Miyakoshi, Kosuke ... [et al]. Effect of different trunk postures on scapular muscle activities and kinematics during shoulder external rotation. Journal of Shoulder and Elbow Surgery 2019, 28(12): 2438-2446

\section{ISSUE DATE:}

2019-12

URL:

http://hdl.handle.net/2433/244811

\section{RIGHT:}

(c) 2019. This manuscript version is made available under the CC-BY-NC-ND 4 . license

http://creativecommons.org/licenses/by-nc-nd/4.0/; The full-text file will be made open to the public on 1 December 2020 in accordance with publisher's 'Terms and Conditions for Self-Archiving'; この論文は出版社版でありません。引用 の際には出版社版をご確認じ利用ください。; This is not the published version. Please cite only the published version. 
Scapular angles with different trunk postures

1

\section{Title page}

2 Effect of different trunk postures on scapular muscle activities and kinematics during shoulder

3 external rotation

4

5 Kosuke Miyakoshi, MS ; Jun Umehara, PhD, PT ${ }^{\mathrm{a}, \mathrm{b}}$; Tomohito Komamura, MS, PT ; Yasuyuki Ueda,

$6 \quad$ PhD, PT ${ }^{\mathrm{a}, \mathrm{c}}$; Toru Tamezawa, MS, $\mathrm{PT}^{\mathrm{d}}$; Gakuto Kitamura, MS, $\mathrm{PT}^{\mathrm{e}}$; Noriaki Ichihashi, PhD, $\mathrm{PT}^{\mathrm{a}}$

$8 \quad{ }^{a}$ Human Health Science, Graduate School of Medicine, Kyoto University

$9 \quad$ besearch fellow of the Japan Society for the Promotion of Science

${ }^{\mathrm{c}}$ Department of Rehabilitation, Nobuhara Hospital

${ }^{\mathrm{d} D e p a r t m e n t ~ o f ~ R e h a b i l i t a t i o n, ~ K a n a z a w a ~ U n i v e r s i t y ~ H o s p i t a l ~}$

${ }^{\mathrm{e}}$ Rehabilitation Unit, Kyoto University Hospital

*Corresponding author

Kosuke Miyakoshi

Human Health Sciences, Graduate School of Medicine, Kyoto University, 
Scapular angles with different trunk postures

E-mail: miyakoshi.kosuke.87n@st.kyoto-u.ac.jp

Financial biases: None

21

22

Ethics committee approval

23 The study design was approved by the ethic committee of Kyoto University Graduate School and the

Faculty of Medicine (C1247-1).

25

26

Acknowledgments: None

27 
Scapular angles with different trunk postures

\section{Abstract}

29 [Background]

30 Shoulder external rotation at abduction (ER) is a notable motion in overhead sports

31 because it could cause strong stress to the elbow and shoulder joint. However, no study

32 has comprehensively investigated the effect of different trunk postures during ER. This

33 study aimed to investigate the effect of different trunk postures on scapular kinematics

34 and muscle activities during ER.

35 [Methods]

$36 \quad$ Fourteen healthy men performed active shoulder external rotation at $90 \square$ of abduction

37 with the dominant arm in 15 trunk postures. At maximum shoulder external rotation in

3815 trunk postures, including 4 flexion-extension, 6 trunk rotation, and 4 trunk

39 side-bending postures, as well as upright posture as a control, scapular muscle activities

40 and kinematics were recorded using surface electromyography and an electromagnetic

41 tracking device, respectively. The data obtained in the flexion-extension, trunk rotation,

42 and trunk side-bending postures were compared with those obtained in the upright

43 posture.

44 [Results]

45 In the flexion-extension condition, scapular posterior tilt and external rotation 
Scapular angles with different trunk postures

Scapula; muscle activity; kinematics; trunk posture; shoulder external rotation; exercise.

significantly decreased, but the muscle activities of the lower trapezius and infraspinatus significantly increased in maximum trunk flexion. Moreover, scapular upward rotation and the activity of the serratus anterior significantly increased in maximum trunk extension. In the rotation condition, scapular posterior tilt and external rotation significantly decreased, but the activity of the serratus anterior significantly increased in the maximum contralateral trunk rotation posture. In the trunk side-bending condition, scapular posterior tilt and the external rotation angle significantly decreased.

[Conclusion]

Trunk postures affected scapular kinematics and muscle activities during ER. Our results suggest that different trunk postures activate the lower trapezius and serratus anterior, which induce scapular posterior tilt.

\section{Level of evidence}

Basic Science Study; Kinesiology 
Scapular angles with different trunk postures

64

65

\section{Introduction}

Shoulder joint motion is the harmonious motion by the scapula, humerus, clavicle, and rib cage. In shoulder motion, the role of the scapula is especially important because nonoptimal scapular motion leads to increased stress on peripheral soft tissues of the shoulder joint and could induce shoulder dysfunction and pain. $3,12,18,20,22,36$ Therefore, it is important to focus on the muscle controlling scapular motion. Some studies have suggested that the upper trapezius (UT), lower trapezius (LT), and serratus anterior (SA) muscles coordinately work as a force couple in arm elevation to upwardly rotate the scapula. $6,7,13,15,19,20$

The effect of trunk posture on scapular motion and muscle activity has also been studied. ${ }^{16,29,39}$ Yamauchi et $\mathrm{al}^{39}$ reported that maximum ipsilateral trunk rotation increased the activity of the middle trapezius (MT) and LT muscles and posterior tilt of the scapular angle in arm elevation. However, the investigated trunk postures were limited (eg, trunk ipsilateral rotation or trunk extension). Therefore, our study reports the effects of comprehensive trunk flexion, extension, bilateral side-bending, and bilateral rotation postures during shoulder external rotation at shoulder abduction. Moreover, we sought to investigate the effects of the degree of the trunk angle on scapular kinematics and muscle activity. 
Scapular angles with different trunk postures

Arm elevation motion has often been selected to evaluate scapular muscle activity and kinematics. ${ }^{16,29,39}$ However, overhead sports players frequently perform motions with shoulder external rotation at abduction (ER) with different trunk postures. Some previous studies reported scapular kinematics during overhead sports, ${ }^{26,30,31}$ and one study described that the scapula posteriorly tilts, externally rotates, and rotates upward at shoulder external rotation during baseball pitching. ${ }^{25}$ Moreover, the scapular muscles stabilize the scapula, and an imbalance of these muscles might contribute to injury risk. ${ }^{11}$ In pitching, the shoulder abduction angle from foot strike to release is approximately $90^{\circ}{ }^{8,37}$ Therefore, shoulder external rotation is commonly measured at $90^{\circ}$ of abduction in baseball players ${ }^{4,27,37}$ which may be a position that reflects the scapular kinematics during pitching. Giving the overhead motion, accordingly, the assessment of scapular muscle activities and kinematics during shoulder ER is necessary.

The scapular motions during ER are upward rotation, external rotation, and posterior tilt. ${ }^{23,33}$ The UT, LT, and SA muscles work to upwardly rotate the scapula during ER. ${ }^{10,28}$ In addition, previous studies have reported that the LT and SA muscles work to posteriorly tilt the scapula during arm elevation. ${ }^{21,24}$ It is assumed that these muscles have an important role in scapular kinematics during ER because the scapula is rotated 
Scapular angles with different trunk postures

100 upward, externally rotated, and posteriorly tilted and these muscle activities increase

101 during the given conditions.

102 Examination of the effect of trunk posture on scapular kinematics and muscle activity

103 during shoulder external rotation is crucial during overhead sports activity. The purpose

104 of this research was to evaluate the effects of the difference in trunk posture on scapular

105 kinematics and muscle activity during ER. Trunk extension or ipsilateral rotation has

106 been shown to increase scapular posterior tilt, the external rotation angle, and LT muscle

107 activity during shoulder flexion. ${ }^{16,29,39}$ We hypothesized that the scapular posterior tilt

108 and external rotation angles and the activity of the SA and LT muscles, which contribute

109 to scapular posterior tilt, would increase with trunk extension and ipsilateral rotation

110 during ER. 
Scapular angles with different trunk postures

\section{Material and Methods}

\subsection{Subjects}

A controlled experimental study was conducted. Fourteen healthy men (mean age, $24.2 \pm 1.9$ years) without orthopedic or nervous system disease of the upper limb or trunk were included in the study. All subjects provided consent after receiving written and oral explanations regarding the study. This study conformed to the principles of the Declaration of Helsinki. The sample size was based on a 1-way analysis of variance (ANOVA) with repeated measures (effect size of $0.25, \alpha$ error of .05 , and power of 0.8 ) by use of G*Power (version 3.1; Heinrich Heine University, Dusseldorf, Germany)

122 before the recruitment of subjects. On the basis of the calculation results, the sample size required was 13; this study thus met the statistical power requirement.

\subsection{Experimental procedure}

Scapular kinematics and muscle activity at ER measured in 14 trunk postures were scapular angles, muscle activities, and shoulder external rotation angles were measured 
Scapular angles with different trunk postures

131 and the pelvis not fixed during the task. This posture of the feet and pelvis was the same

132 in all testing postures, and only the trunk posture was changed during the task. Subjects

133 performed 15 trunk postures: upright posture as the control posture; 4 trunk

134 flexion-extension conditions (maximum flexion [Flexmax], $20^{\circ}$ of flexion [Flex20], 20

135 of extension [Ext20], and maximum extension [Extmax]); 6 trunk rotation conditions

136 (maximum contralateral rotation [CR $\max$, contralateral rotation of $30^{\circ}$ [CR30],

137 contralateral rotation of $15^{\circ}$ [CR15], ipsilateral rotation of $15^{\circ}$ [IR15], ipsilateral

138 rotation of $30^{\circ}$ [IR30], and maximum ipsilateral rotation [IR $\max$ ]); and 4 trunk

139 side-bending conditions (contralateral lateral bending at $30^{\circ}$ [CLB30], contralateral

140 lateral bending at $15^{\circ}$ [CLB15], ipsilateral lateral bending at $15^{\circ}$ [ILB15], and ipsilateral

141 lateral bending at $30^{\circ}$ [ILB30]). Three optical markers were attached to the seventh

142 cervical spinous process (C7), 10th thoracic spinous process (T10), and third lumbar

143 spinous process (L3). The flexion-extension angle was made by the line connecting C7

144 with T10 and the line connecting L3 with T10 in the sagittal plane. In the upright

145 posture, the angle was $0^{\circ}$. Flexmax was the posture in which each subject achieved the

146 maximum trunk flexion angle by relaxing. The flexion angle for Flexmax in all subjects

147 was over $20^{\circ}$. The trunk rotation angle was the angle between the line linking the 
Scapular angles with different trunk postures

149 trunk side-bending angle was the angle between the line linking C7 and T10 and the line

150 linking L3 and T10 in the coronal plane.

\subsection{Active shoulder external rotation task}

153 Subjects performed the active ER task to the maximum shoulder external rotation angle

154 with random trunk postures directed from 12 trunk postures except $\mathrm{CR}^{\max }$, IR ${ }^{\max }$, and

155 Ext $^{\max }$ (Fig. 1). Then, they performed the active ER task with randomly directed trunk

156 postures from the remaining 3 trunk postures. Before measurement of scapular

157 kinematics and muscle activity during the shoulder external rotation task, the active maximum shoulder external rotation angle was measured using a goniometer at $90^{\circ}$ of abduction of the shoulder joint in the directed trunk posture. Subsequently, subjects actively maintained the maximum shoulder external rotation position for 5 seconds. The measurement was performed once in each trunk posture to avoid the effect of fatigue.

\subsection{EMG protocol}

During the shoulder external rotation task, scapular muscle activities were collected using surface electromyography (EMG) (TeleMyo 2400; Noraxon, Scottsdale, AZ, 
Scapular angles with different trunk postures

167 infraspinatus, and latissimus dorsi (LD) in the dominant upper limb with fixed 2.5-cm spacing parallel to the muscle fibers. Skin at the electrode sites was shaved and cleaned using scrubbing gel and alcohol. Electrode placement was based on previous studies or recommendations. The locations of the electrodes for each muscle were as follows: The UT electrode is at the midpoint between C7 and the acromion of the scapula. ${ }^{19}$ The MT electrode is at the midpoint between the medial border of the scapula and T3. The LT electrode is at the point located at two-thirds on the line from the trigonum spinae (TS) to T8. The infraspinatus electrode is at the midpoint on the line connecting the midpoint of the spine of the scapula and angulus inferior scapulae.14 The SA electrode is at the halfway point between the anterior border of the LD muscle and the inferior border of the pectoralis major muscle on the seventh rib. ${ }^{9}$ The LD electrode is 2 to $3 \mathrm{~cm}$ below the angulus inferior scapulae. ${ }^{32}$ The raw EMG signals during the shoulder external rotation task were recorded and analyzed for 3 seconds at the shoulder maximum external rotation angle. The EMG signals of the maximal voluntary contraction were recorded for 3 seconds on each muscle. The method was referred to the manual muscle test and previous studies $^{2,5,17,32}$ before subjects began the task. The raw EMG signals were band pass filtered (15-500 Hz, Butterworth) and then smoothed using the root mean square. 
Scapular angles with different trunk postures

185 The root-mean- square amplitude was divided by the maximal voluntary contraction of each muscle for normalization.

\subsection{Scapular kinematics}

189 Three-dimensional kinematics of the scapula and thorax was quantified during the

190 shoulder external rotation task using a 6-df electromagnetic tracking device (Liberty;

191 Polhemus, Colchester, VT, USA) at $120 \mathrm{~Hz}$. This system was composed of a transmitter,

1925 sensors, and a digitizing stylus connecting the Liberty electronic unit. The transmitter

193 was fixed on a rigid wooden stand at $100 \mathrm{~cm}$ in height. This transmitter generated the

194 electromagnetic fields, which constituted the global coordinate system, with the x-axis

195 orienting forward, the y-axis orienting upward, the z-axis orienting right, and the origin

196 located at the transmitter. The sensors were placed on the bony landmarks of the

197 subjects using tape. The thoracic sensor was placed at the sternum just below the jugular notch; the humeral sensor, on the halfway point of the humerus with a thermoplastic cuff; and the scapular sensor, on the flat surface of the acromion. With reference to the positions of these sensors, the local coordinate systems (LCSs) of the thorax, humerus, and scapula were built by digitizing each bony landmark while subjects sat in the anatomic upper-limb position. 
Scapular angles with different trunk postures

All LCSs were defined according to the shoulder standardization proposal of the

International Society of Biomechanics. ${ }^{38}$ The distal coordinate system was rotated with

respect to the proximal coordinate system in accordance with the recommendation on

207 the origin was the acromial angle (AA). The axes were defined as follows: The $\mathrm{x}$-axis

208 (Xs) was the normal vector of the plane including the TS, AA, and inferior angle. The

209 z-axis (Zs) was directed from the TS to the AA. The y-axis (Ys) was the normal vector

210 of the x-axis and z-axis. In the LCS of the thorax, the origin was the sternal notch. The

$211 \mathrm{y}$-axis (Yt) was directed from the midpoint between the xiphoid process and T8 to the

212 midpoint between the SN and C7. The $\mathrm{z}$-axis $(\mathrm{Zt})$ was the normal vector of the plane

213 including the midpoint between the xiphoid process and T8, SN, and C7. The direction

214 was right. The $\mathrm{x}$-axis $(\mathrm{Xt})$ was the normal vector of the $\mathrm{y}$-axis and $\mathrm{z}$-axis.

216 Xt was defined as right $(+)$ and left $(-)$ bending, that around Yt was defined as rotation

217 to the left $(+)$ and rotation to the right $(-)$, and that around $\mathrm{Zt}$ was defined as extension

$218(+)$ and flexion (-). The rotation of the scapular segment relative to the thoracic segment around Xs was defined as downward $(+)$ and upward (-) rotation, that around Ys was 
Scapular angles with different trunk postures

221

222

223

224

225

226

227

228

229

230

231

232

233

234

235

236

237

posterior (+) and anterior (-) tilt.

The humeral external rotation angle was defined as the difference between the apparent shoulder external rotation angle measured by a goniometer and the thoracic extension angle and scapular posterior tilt angle. The scapular angle of each trunk posture was the average of kinematic data for 3 seconds at the shoulder maximum external rotation angle.

\subsection{Data analysis}

The statistical analysis software used in this study was SPSS, version 22 (IBM, Armonk, NY, USA). For the scapular angle and muscle activity, 1-way ANOVA with repeated measures on 1 factor (trunk posture) was used to evaluate the effect of trunk posture on each parameter. Then, trunk postures were classified into 4 conditions: upright as the control condition, flexionextension condition (Flexmax, Flex20, Ext20, and Extmax), rotation condition (IR15, IR30, IRmax, CR15, CR30, and CRmax), and side-bending condition (CLB30, CLB15, ILB15, and ILB30). For the scapular angle and muscle activity, 1-way ANOVA with repeated measures on a factor (trunk posture) was used in each condition including upright posture. When a significant main effect was detected, the Dunnett test as the post hoc test was conducted to compare the trunk 
Scapular angles with different trunk postures

postures with the upright posture.

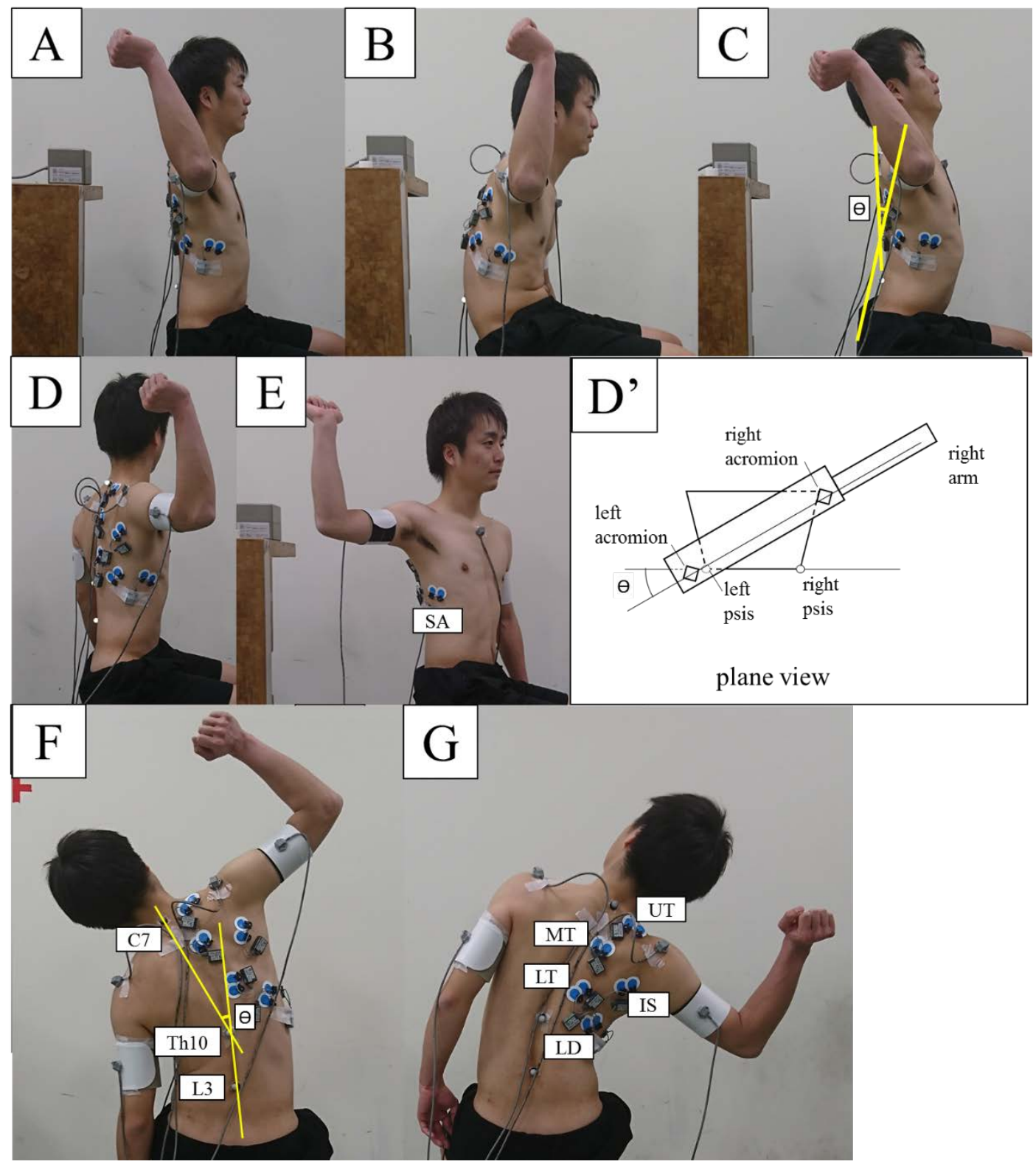

241 Figure 1 Different trunk postures during 2nd ER.

242 Participants performed shoulder external rotation at shoulder $90^{\circ}$ abduction with 243 different trunk postures. Electromyography electrodes were placed on UT (upper 244 trapezius muscle), MT (middle trapezius muscle), LT (Lower trapezius muscle), SA 245 (serratus anterior), IS (infraspinatus muscle), and LD (latissimus dorsi). Three optical 246 markers were attached to the 7th cervical spinous process (C7), 10th thoracic spinous 247 process (Th10), and 3rd lumbar spinous process (L3). $\theta$ means contralateral lateral 248 bending angle. A: upright posture. B: Flexion posture. C: Extension posture. D: 
Scapular angles with different trunk postures

249 Contralateral rotation. E: Ipsilateral rotation. F: Contralateral lateral bending. G: 250 Ipsilateral lateral bending. D' shows trunk rotation angle defined by the line linking the 251 bilateral acromion and the line linking the bilateral posterior anterior iliac spine (psis). $\theta$, 252 contralateral lateral bending angle. 
Scapular angles with different trunk postures

Table I. Kinematics data

\begin{tabular}{|c|c|c|c|c|c|}
\hline & & $\mathrm{GH}\left({ }^{\circ}\right)$ & & Scapula $\left(^{\circ}\right)$ & \\
\hline & Posture & External rotation & Posterior tilt & Upward rotation & Internal rotation \\
\hline Control & Upright & $89 \pm 14$ & $13 \pm 6$ & $10 \pm 10$ & $12 \pm 7$ \\
\hline \multirow{5}{*}{$\begin{array}{l}\text { Flexion and } \\
\text { Extension }\end{array}$} & Flex max & $83 \pm 16[.534]$ & $7 \pm 8 *[.000]$ & $12 \pm 11[.078]$ & $19 \pm 6 *[.001]$ \\
\hline & Flex20 & $84 \pm 12$ [.607] & $10 \pm 7[.103]$ & $9 \pm 10[.650]$ & $14 \pm 5[.722]$ \\
\hline & (Ext20) & $(88 \pm 10)$ & $(12 \pm 9)$ & $(13 \pm 11)$ & $(13 \pm 10)$ \\
\hline & Ext max & $97 \pm 11$ [.259] & $12 \pm 6[.591]$ & $12 \pm 12 *[.027]$ & $10 \pm 6[.347]$ \\
\hline & Main effect & $\mathrm{F}=3.26, \mathrm{p}=.029$ & $\mathrm{~F}=8.66, \mathrm{p}<.001$ & $\mathrm{~F}=3.46, \mathrm{p}=.026$ & $\mathrm{~F}=9.79, \mathrm{p}<.001$ \\
\hline \multirow{7}{*}{ Rotation } & $\mathrm{CR}_{\max }$ & $79 \pm 11 *[.001]$ & $8 \pm 7 *[.000]$ & $10 \pm 11$ & $17 \pm 6 *[.001]$ \\
\hline & CR30 & $82 \pm 10 *[.020]$ & $11 \pm 6$ [ .059] & $11 \pm 10$ & $14 \pm 5[.102]$ \\
\hline & CR15 & $87 \pm 12[.836]$ & $11 \pm 6[.122]$ & $10 \pm 10$ & $15 \pm 5 *[.046]$ \\
\hline & IR15 & $91 \pm 10$ [ 1.000$]$ & $14 \pm 6[.615]$ & $11 \pm 10$ & $13 \pm 6[.659]$ \\
\hline & IR30 & $90 \pm 12[.980]$ & $14 \pm 6[.287]$ & $11 \pm 11$ & $14 \pm 6[.447]$ \\
\hline & $I R$ max & $88 \pm 17[.991]$ & $13 \pm 6[1.000]$ & $11 \pm 12$ & $12 \pm 7[1.000]$ \\
\hline & Main effect & $\mathrm{F}=6.57, \mathrm{p}<0.001$ & $\mathrm{~F}=14.74, \mathrm{p}<.001$ & $\mathrm{~F}=1.36, \mathrm{p}=.241$ & $\mathrm{~F}=3.75, \mathrm{p}=.002$ \\
\hline \multirow{5}{*}{$\begin{array}{l}\text { Lateral } \\
\text { bending }\end{array}$} & ILB30 & $90 \pm 10$ & $9 \pm 6 *[.015]$ & $11 \pm 10[.979]$ & $15 \pm 6[.170]$ \\
\hline & ILB15 & $85 \pm 11$ & $11 \pm 6[.456]$ & $11 \pm 11[.925]$ & $14 \pm 6[.407]$ \\
\hline & CLB15 & $86 \pm 13$ & $9 \pm 7 *[.018]$ & $11 \pm 10[.879]$ & $18 \pm 7 *[.001]$ \\
\hline & CLB30 & $90 \pm 11$ & $7 \pm 7 *[.001]$ & $10 \pm 9[.996]$ & $16 \pm 5 *[.018]$ \\
\hline & Main effect & $\mathrm{F}=1.86, \mathrm{p}=.132$ & $\mathrm{~F}=4.62, \mathrm{p}=.003$ & $\mathrm{~F}=0.36, \mathrm{p}=.838$ & $\mathrm{~F}=4.68, \mathrm{p}=.003$ \\
\hline
\end{tabular}


Scapular angles with different trunk postures

GH, glenohumeral; Flexmax, maximum flexion; Flex20, $20^{\circ}$ of flexion; Ext20, 20 of extension; Ext $t_{\max }$ maximum extension; $\mathrm{CR}_{\max }$ maximum contralateral rotation; CR30, contralateral rotation of $30^{\circ}$; CR15, contralateral rotation of $15^{\circ}$; IR15, ipsilateral rotation of $15^{\circ}$; IR30, ipsilateral rotation of $30^{\circ}$; $\mathrm{IR}_{\max }$, maximum ipsilateral rotation; CLB30, contralateral lateral bending at $30^{\circ}$; CLB15, contralateral lateral bending at 15; ILB15, ipsilateral lateral bending at 15; ILB30, ipsilateral lateral bending at $30^{\circ}$; F, Fishers value.

Data are presented as mean \pm standard deviation. The P value for each value is shown in brackets.

* Significantly different $(\mathrm{P}<.05)$ compared with upright posture.

†The Ext20 values were not included in the analysis because only 5 subjects achieved Ext20. The values are shown as reference values. 
Scapular angles with different trunk postures

283

\begin{tabular}{|c|c|c|c|c|c|c|c|}
\hline \multicolumn{8}{|c|}{ Muscle activation (\%MVC) } \\
\hline & Muscle & UT & MT & $\mathrm{LT}$ & IS & SA & LD \\
\hline Control & Upright & $15.9 \pm 13.4$ & $23.3 \pm 15.3$ & $30.3 \pm 18.9$ & $40.7 \pm 30.2$ & $27.1 \pm 16.6$ & $6.6 \pm 5.0$ \\
\hline \multirow{5}{*}{$\begin{array}{l}\text { Flexion and } \\
\text { Extension }\end{array}$} & Flex max & $18.7 \pm 13.1[.160]$ & $32.1 \pm 15.8$ & $45.7 \pm 28.3 *[.027]$ & $54.5 \pm 41.2 *[.019]$ & $22.2 \pm 9.1[.953]$ & $5.7 \pm 4.0$ \\
\hline & Flex20 & $19.7 \pm 12.5[.069]$ & $29.0 \pm 15.4$ & $43.0 \pm 28.2[.090]$ & $50.6 \pm 38.4[.125]$ & $25.0 \pm 10.8[.554]$ & $6.5 \pm 4.3$ \\
\hline & $($ Ext20) & $(13.3 \pm 18.2)$ & $(10.9 \pm 11.0)$ & $(15.0 \pm 15.8)$ & $(35.1 \pm 27.9)$ & $(29.0 \pm 16.0)$ & $(9.6 \pm 10.8)$ \\
\hline & Ext max & $14.6 \pm 11.5[.957]$ & $25.1 \pm 19.1$ & $27.0 \pm 16.8[.937]$ & $45.9 \pm 31.3[.633]$ & $42.5 \pm 23.8 *[.006]$ & $7.4 \pm 4.2$ \\
\hline & Main effect & $\mathrm{F}=3.63, \mathrm{p}=.021$ & $\mathrm{~F}=1.86, \mathrm{p}=.153$ & $\mathrm{~F}=4.93, \mathrm{p}=.005$ & $\mathrm{~F}=3.04, \mathrm{p}=.040$ & $\mathrm{~F}=3.47, \mathrm{p}=.014$ & $\mathrm{~F}=1.63, \mathrm{p}=.199$ \\
\hline \multirow{7}{*}{ Rotation } & $\mathrm{CR}_{\max }$ & $16.4 \pm 13.7[.999]$ & $23.1 \pm 15.0[1.000]$ & $18.7 \pm 10.1[.349]$ & $53.4 \pm 31.0$ & $36.3 \pm 15.9 *[.003]$ & $7.9 \pm 4.3$ \\
\hline & CR30 & $16.2 \pm 11.7[1.000]$ & $21.5 \pm 13.2[.991]$ & $25.1 \pm 13.3[.945]$ & $50.4 \pm 42.8$ & $31.1 \pm 19.1[.863]$ & $7.4 \pm 5.2$ \\
\hline & CR15 & $16.6 \pm 12.8[.651]$ & $23.5 \pm 16.8[1.000]$ & $26.2 \pm 15.6$ [.999] & $42.9 \pm 32.1$ & $27.4 \pm 11.0[1.000]$ & $7.0 \pm 4.9$ \\
\hline & IR15 & $18.1 \pm 15.2[.597]$ & $29.9 \pm 19.2$ [.375] & $44.1 \pm 28.3[.172]$ & $44.3 \pm 29.4$ & $27.3 \pm 10.5[1.000]$ & $6.3 \pm 4.6$ \\
\hline & IR30 & $20.1 \pm 15.1$ [.155] & $33.8 \pm 25.7 *[.020]$ & $48.2 \pm 30.8 *[.050]$ & $46.4 \pm 36.9$ & $28.1 \pm 14.3[1.000]$ & $6.5 \pm 4.0$ \\
\hline & $I_{\text {max }}$ & $21.7 \pm 17.2 *[.015]$ & $31.6 \pm 20.9$ [.117] & $49.5 \pm 33.6 *[.025]$ & $40.8 \pm 26.1$ & $25.1 \pm 12.3[.996]$ & $6.2 \pm 4.2$ \\
\hline & Main effect & $\mathrm{F}=2.48, \mathrm{p}=.030$ & $\mathrm{~F}=20.77, \mathrm{p}<.001$ & $\mathrm{~F}=6.58, \mathrm{p}<.001$ & $\mathrm{~F}=2.00, \mathrm{p}=.076$ & $\mathrm{~F}=3.92, \mathrm{p}=.002$ & $F=2.08, p=.065$ \\
\hline \multirow{5}{*}{$\begin{array}{l}\text { Lateral } \\
\text { bending }\end{array}$} & ILB30 & $15.1 \pm 12.4$ & $26.4 \pm 13.2[.862]$ & $27.0 \pm 18.3$ & $39.7 \pm 30.4$ [.997] & $35.2 \pm 17.5[.153]$ & $5.9 \pm 3.7$ \\
\hline & ILB15 & $14.0 \pm 10.9$ & $26.9 \pm 19.3[.831]$ & $31.0 \pm 21.5$ & $38.4 \pm 24.5[.968]$ & $36.8 \pm 18.1[.471]$ & $5.9 \pm 3.7$ \\
\hline & CLB15 & $22.2 \pm 19.8$ & $31.1 \pm 16.1[.250]$ & $32.1 \pm 19.2$ & $51.5 \pm 37.0[.109]$ & $19.4 \pm 9.5[.680]$ & $5.2 \pm 2.6$ \\
\hline & CLB30 & $22.8 \pm 26.2$ & $39.3 \pm 20.0 *[.006]$ & $37.3 \pm 26.7$ & $53.5 \pm 36.9 *[.044]$ & $22.0 \pm 23.7[.891]$ & $4.6 \pm 2.3$ \\
\hline & Main effect & $F=1.90, p=.124$ & $\mathrm{~F}=3.20, \mathrm{p}=.020$ & $\mathrm{~F}=0.79, \mathrm{p}=.539$ & $\mathrm{~F}=4.20, \mathrm{p}=.005$ & $\mathrm{~F}=3.47, \mathrm{p}=.014$ & $F=1.80, p=.143$ \\
\hline
\end{tabular}

Table II. EMG data 
Scapular angles with different trunk postures

MVC, maximal voluntary contraction; UT, upper trapezius muscle; MT, middle trapezius muscle; LT, lower trapezius muscle; IS, infraspinatus muscle; SA, serratus anterior; LD, latissimus dorsi; Flexmax, maximum flexion; Flex20, 20 of flexion; Ext20, 20ºf extension; Extmax, maximum extension; $\mathrm{CR}_{\max }$, maximum contralateral rotation; CR30, contralateral rotation of $30^{\circ}$; CR15, contralateral rotation of $15^{\circ}$; IR15, ipsilateral rotation of $15^{\circ}$; IR30, ipsilateral rotation of $30^{\circ}$; IRmax, maximum ipsilateral rotation; CLB30, contralateral lateral bending at $30^{\circ}$; CLB15, contralateral lateral bending at $15^{\circ}$; ILB15, ipsilateral lateral bending at $15^{\circ}$; ILB30, ipsilateral lateral bending at $30^{\circ}$; F, Fishers value. Data are presented as mean \pm standard deviation. The $\mathrm{P}$ value for each value is shown in brackets.

* Significantly different $(\mathrm{P}<.05)$ compared with upright posture.

$\dagger$ The Ext20 values were not included in the analysis because only 5 subjects achieved Ext20. The values are shown as reference values. 
Scapular angles with different trunk postures

\section{Results}

All subjects achieved the rotation and side-bending conditions. However, only 5

subjects performed the Ext20 task, and another subject performed Extmax at a trunk angle of less than $20^{\circ}$ of extension. Therefore, the data are shown as reference values but were not included in the analysis. The maximum trunk angle in each trunk condition was $37^{\circ} \pm 6_{\text {_ }}$ for maximum trunk flexion, $14^{\circ} \pm 8^{\circ}$ for maximum trunk extension, $44^{\circ} \pm 8^{\circ}$ for maximum contralateral trunk rotation, and $42^{\circ} \pm 7^{\circ}$ for maximum ipsilateral trunk rotation. The kinematic data of 1 subject for Extmax were excluded because of measurement failure. The kinematic and muscle activity data are described in the following sections.

\subsection{Kinematics data}

indicated a main effect in all conditions for the angle of glenohumeral joint external rotation. The post hoc test revealed that the angle of external rotation in CRmax and CR30 significantly decreased compared with that in the upright posture. For scapular posterior tilt, a main effect in all conditions was shown. Scapular posterior tilt in 
Scapular angles with different trunk postures

312 Flexmax, CR $\max$, ILB30, CLB15, and CLB30 significantly decreased compared with

313 that in the upright posture. For the angle of scapular upward rotation, a main effect was

314 shown in the flexion-extension condition only. The scapula in Extmax was slightly

315 upwardly rotated compared with that in the upright posture. For the scapular external

316 rotation angle, a main effect was shown in all conditions. The angle in Flexmax, $\mathrm{CR}_{\max }$,

317 CR15, CLB15, and CLB30 significantly decreased.

\subsection{Muscle activity data}

320

All muscle activities are presented in Table II. In the UT, 1- way ANOVA showed

a main effect in the flexion-extension and rotation conditions. The muscle activity in

IRmax significantly increased compared with that in the upright posture. In the MT, a

main effect was shown in the rotation and side-bending conditions. The muscle activity

in IR30 and CLB30 significantly increased compared with that in the upright posture.

In the LT, a main effect was shown in the flexion-extension and rotation conditions.

The muscle activity significantly increased in Flexmax, IR30, and IRmax. In the

infraspinatus, a main effect was shown in the flexion-extension and side-bending

conditions. The muscle activity in Flexmax and CLB30 significantly increased 
Scapular angles with different trunk postures

330

331

332

333

334

335

336

conditions. Extmax and CRmax increased the muscle activity more significantly than the upright posture. In the LD, there were no main effects in all conditions.

\section{Discussion}

In this study, we examined the effect of trunk posture on scapular kinematics and muscle activity at maximum shoulder external rotation. To our knowledge, this is the first research study to demonstrate that flexion, extension, rotation, and lateral bending of the trunk minimize the effects of hip motions on scapular kinematics and muscle activity. We hypothesized that extension or ipsilateral rotation of the trunk would contribute to increases in the scapular posterior tilt angle, external rotation angle, and activities of the SA and LT, which are the posterior tilt muscles of the scapula. Our results showed that the scapular posterior tilt angle did not change whereas the SA and LT activities increased with trunk extension and $\mathrm{IR}_{\max }$, respectively. It was assumed that this upright posture was relatively close to extension of the trunk considering that only a few subjects achieved trunk extension over $20^{\circ}$. In addition, there were no trunk postures in which both LT and SA activities increased.

In the trunk flexion-extension condition, the angles of scapular posterior tilt and external rotation significantly decreased in Flexmax compared with those in the upright 
Scapular angles with different trunk postures

348 posture during ER. Kebaetse et $\mathrm{al}^{16}$ reported that shoulder abduction range of motion

349 and the angle of scapular upward rotation and posterior tilt during arm elevation

350 decreased with a slouch posture. In addition, they indicated that the acromion may

351 create a bony block that may cause or contribute to impingement pathology with

352 repetitive overhead activity. Our study similarly indicated a decrease in the scapular

353 posterior tilt angle with trunk flexion, which could also cause a bony block. The angle

354 of scapular external rotation decreased whereas the angle of scapular upward rotation

355 did not change in Flexmax compared with that in the upright posture - a finding that

356 was partially incongruent with the results of Kebaetse et al. This is considered to be

357 due to the difference in examination posture; their study was not on ER but rather on

358 arm elevation. In Extmax in our study, the angle of scapular upward rotation and the

359 activity of the SA significantly increased compared with those in the upright posture,

360 which is logical considering that the SA has the function of scapular upward

361 rotation. ${ }^{10,28}$ The difference of approximately $2^{\circ}$ in the scapular upward rotation angle

362 between the upright posture and Extmax is small. Nonetheless, Shaheen et al ${ }^{34}$ reported that rigid and elastic taping techniques changed the scapular internal rotation and posterior tilt angles by less than $5^{\circ}$ and reduced pain in patients with shoulder impingement syndrome. Therefore, the change of $2^{\circ}$ maximum with extension may be 
Scapular angles with different trunk postures

366 clinically significant. We assumed that the differences between the Extmax and upright

367 postures were not enough for some subjects to increase the angle of scapular tilt in

368 Extmax compared with that in the upright posture.

In the trunk rotation condition, the angles of scapular posterior tilt and

external rotation significantly decreased in $\mathrm{CR}_{\max }$ compared with those in the upright

posture. Scapular external rotation significantly decreased in CR15 compared with that

372 in the upright posture, whereas in $\mathrm{CR}_{\max }$ and CR30, the glenohumeral joint external rotation angle significantly decreased. This restriction of shoulder external rotation is

predictably caused by the stretched LD, which contributes as a shoulder internal rotator, has the origin at the spine and pelvis, and inserts in the humerus. ${ }^{1}$ In IR30 and IRmax, the angle of scapular upward rotation did not significantly increase whereas the activity of the LT on scapular upward rotation significantly increased. The increase in LT activity without an increment in scapular upward rotation could be evoked by the physical restriction of the scapular motion by the thorax or the increase in activity of the scapular downward rotators such as the rhomboids, ${ }^{10}$ which was not measured in this study. 
Scapular angles with different trunk postures

384 This study showed no significant differences in scapular kinematics whereas UT and

385 LT activities significantly increased. The methodology regarding posture differed

386 between our study and this previous study. Subjects performed our task in the sitting

387 position because the purpose of this study was to investigate the effects of trunk

388 posture only. In the study by Yamauchi et al, subjects performed active ER in the

389 standing position; therefore, their study included pelvis rotation. In addition, the

390 upright posture in our study was relatively in a trunk-extended posture. It was assumed

391 that the variance of the results was caused by the definition of postures.

392 In the side-bending condition, the angles of scapular posterior tilt and external rotation

393 significantly decreased in CLB30 compared with those in the upright posture. In

394 CLB15, only the scapular external rotation angle significantly decreased. It was considered that trunk contralateral bending disturbed scapular external rotation and that MT activity compensatively increased to resist it. In ILB30, the angle of scapular posterior tilt significantly decreased compared with that in the upright posture.

The low activity in the muscles could cause the decrease in scapular posterior tilt.

However, there were no decreases in the activities of the LT and SA- the posterior tilt muscles- in trunk postures that showed a significant decrease in the scapular posterior 
Scapular angles with different trunk postures

402 the alteration in scapular muscle activities. The trunk posture was only the factor that

403 differed among these conditions. Consequently, it was considered that the thorax

404 physically restricted the scapular movement, resulting in a decrease in the scapular

405 posterior tilt angle. Moreover, the trunk postures that decreased the angle of scapular

406 external rotation roughly duplicated the trunk postures in which the scapular posterior

407 tilt angle decreased. The decrease in the scapular external rotation angle might also be

408 due to the scapular movement restriction by the thorax.

409 Our hypothesis was that the activities of the LT and SA that contribute to scapular

410 posterior tilt would synchronously change with it. However, the increase or decrease in

411 the activities of the 2 muscles did not happen simultaneously. On the contrary, the

412 activity of 1 muscle tended to increase in a certain trunk posture while the activity of

413 the other decreased in the same trunk posture. These results suggested that there was a

414 superiority among muscles that have similar action, which may be replaced based on

415 the difference in the trunk posture. These muscle activities might be coordinated to be

416 the most effective muscle force balance for the task because the superiority did not

417 change based on the increase or decrease in the scapular posterior tilt angle.

418 This study has some limitations. First, the trunk postures were uniquely defined

419 based on the body surface markers, although some previous studies used similar angle 
Scapular angles with different trunk postures

420 definitions. ${ }^{27,35}$ Second, the upright posture did not take into account individual

421 specificity. Trunk posture was suggested to be better defined on the basis of the

422 individual trunk range of motion and neutral trunk posture. If the natural trunk posture

423 (neutral trunk posture) was based on the aforementioned definition of trunk posture, all

424 the participants might have achieved Ext20. Finally, surface EMG was not able to

measure the deep muscles. The effects of trunk posture on the deep muscles in the present research are unknown. kinematics during ER, it is suggested to choose a trunk extension posture rather than a trunk flexion posture because the angles of scapular posterior tilt and external rotation

430 decreased during the task of ER with Flexmax in this study. In addition, ipsilateral rotation of the trunk increased the scapular posterior tilt angle and LT activity, which is important in ER; therefore, adding ipsilateral rotation to trunk extension is recommended.

Trunk flexion and ipsilateral rotation postures may resist scapular upward rotation.

The activation of the LT with these trunk postures suggests that the LT may be effective for scapular upward rotation in these postures. We suggest that Flexmax, IRmax, and IR30 would facilitate LT activity during shoulder external rotation at 90_ of 
Scapular angles with different trunk postures

438 shoulder abduction. Similarly, Extmax and CRmax would facilitate SA activity during

439 such shoulder exercise. From the perspective of intensive training of those muscles,

440 future studies are needed to research scapular muscle activities at maximum shoulder

441 external rotation torque. 
Scapular angles with different trunk postures

\section{Conclusion}

This study showed that the difference in trunk posture affected scapular kinematics

446 and muscle activity during active shoulder external rotation at $90^{\circ}$ of abduction. The

447 LT and SA, which both contribute to scapular posterior tilt, were activated by different 448 trunk postures. 
Scapular angles with different trunk postures

\section{References}

452

453

454

455

456

457

458

459

460

461

462

463

464

465

466

1. Bassett RW, Browne AO, Morrey BF, An KN. Glenohumeral muscle force and moment mechanics in a position of shoulder instability. J Biomech. 1990;23(5):405-415.

2. Brandt M, Andersen LL, Samani A, Jakobsen MD, Madeleine P. Inter-day reliability of surface electromyography recordings of the lumbar part of erector spinae longissimus and trapezius descendens during box lifting. BMC Musculoskelet Disord. 2017;18(1):519.

https://bmcmusculoskeletdisord.biomedcentral.com/articles/10.1186/s12891-0171872-ydoi:10.1186/s12891-017-1872-y

3. Burkhart SS, Morgan CD, Kibler W Ben. The disabled throwing shoulder: Spectrum of pathology part III: The SICK scapula, scapular dyskinesis, the kinetic chain, and rehabilitation. Arthroscopy. 2003;19(6):641-661. doi:10.1016/S0749-8063(03)00389-X

4. Camp CL, Zajac JM, Pearson DB, Sinatro AM, Spiker AM, Werner BC, et al. Decreased Shoulder External Rotation and Flexion Are Greater Predictors of Injury Than Internal Rotation Deficits: Analysis of 132 Pitcher-Seasons in 
Scapular angles with different trunk postures

Professional Baseball. Arthroscopy. 2017;33(9):1629-1636.

http://dx.doi.org/10.1016/j.arthro.2017.03.025doi:10.1016/j.arthro.2017.03.025

5. Chalmers PN, Trombley R, Cip J, Monson B, Forsythe B, Nicholson GP, et al. Postoperative Restoration of Upper Extremity Motion and Neuromuscular Control During the Overhand Pitch. Am J Sports Med. 2014;42(12):2825-2836. http://journals.sagepub.com/doi/10.1177/0363546514551924doi:10.1177/036354 6514551924

6. Cools AM, Witvrouw EE, Declercq GA, Vanderstraeten GG, Cambier DC. Evaluation of isokinetic force production and associated muscle activity in the scapular rotators during a protraction-retraction movement in overhead athletes with impingement symptoms. Br J Sports Med. 2004;38(1):64-68. doi:10.1136/bjsm.2003.004952

7. Cools AM, Witvrouw EE, Mahieu NN, Danneels L a. Isokinetic Scapular Muscle Performance in Overhead Athletes With and Without Impingement Symptoms. J Athl Train. 2005;40(2):104-110.

8. Dillman CJ FG\& AJ. Biomechanics of Pitching with Emphasis upon Shoulder Kinematics. J Orthop Sports Phys Ther. 1993;18(2):402-408. doi:10.2519/jospt.1993.18.2.402 
Scapular angles with different trunk postures

9. Ekstrom RA, Bifulco KM, Lopau CJ, Andersen CF, Gough JR. Comparing the Function of the Upper and Lower Parts of the Serratus Anterior Muscle Using Surface Electromyography. J Orthop Sports Phys Ther. 2004;34(5):235-243. doi:10.2519/jospt.2004.1345

10. Escamilla RF. Shoulder Muscle Activity and Function in Common Shoulder Rehabilitation Exercise. Sports Med. 2009;39(8):663-685. doi:10.2165/00007256-200939080-00004.

11. Escamilla RF, Andrrews JR. Shoulder muscle recruitment patterns and related biomechanics during upper extremity sports. Sports Med. 2009;39(7):569-590.

12. Graichen H, Bonei H, Stammberger T, Haubner M, Englmeier K.

Three-Dimensional Analysis of the Width of the Subacromial Space in Healthy Subjects and Patients with Impingement Syndrome. AJR Am J Roentgenol. 1999;172(4):1081-1086.

13. Ha S min, Kwon O yun, Cynn H seock, Lee W hwee, Park K nam, Kim S hyun, et al. Comparison of electromyographic activity of the lower trapezius and serratus anterior muscle in different arm-lifting scapular posterior tilt exercises. Phys Ther Sport. 2012;13(4):227-232. http://dx.doi.org/10.1016/j.ptsp.2011.11.002doi:10.1016/j.ptsp.2011.11.002 
Scapular angles with different trunk postures

504

505

506

507

508

509

510

511

512
14. Iles R, Davidson M. Evidence based practice: a survey of physiotherapists' current practice. Physiother Res Int. 2006;11(2):93-103. doi:10.1002/pri

15. Johnson G, Bogduk N, Nowitzke A, House D. Anatomy and actions of the trapezius muscle. Clin Biomech (Bristol, Avon). 1994;9(1):44-50.

doi:10.1016/0268-0033(94)90057-4

16. Kebaetse M, McClure P, Pratt NA. Thoracic position effect on shoulder range of motion strength, and three-dimensional scapular kinematics. Arch Phys Med Rehabil. 1999;80(8):945-950. doi:10.1016/S0003-9993(99)90088-6

17. Kendall FP, McCreary EK, Provanoe PG, Rodgers M RW. Muscles: Testing and Function, with Posture and Pain. 5th ed. Lippincott Williams \& Wilkins; 2005.

18. Kibler W Ben, Sciascia A. Current concepts: scapular dyskinesis. Br J Sports Med.2010 Apr;44(5):300-305. doi:10.1136/bjsm.2009.058834

19. Kibler W Ben, Sciascia AD, Uhl TL, Tambay N, Cunningham T. Electromyographic analysis of specific exercises for scapular control in early phases of shoulder rehabilitation. Am J Sports Med. 2008;36(9):1789-1798. doi:10.1177/0363546508316281

20. Ludewig PM, Cook TM. Alterations in shoulder kinematics and associated muscle activity in people with symptoms of shoulder impingement. Phys Ther. 
Scapular angles with different trunk postures

522

523

524

525

526

527

528

529

530

531

532

533

534

535

536

537

538

539

2000;80(3):276-91.

http://www.ncbi.nlm.nih.gov/pubmed/10696154doi:10.2519/jospt.1993.17.5.212

21. Ludewig PM, Cook TM, Nawoczenski DA. Three-dimensional scapular orientation and muscle activity at selected positions of humeral elevation. $\mathrm{J}$ Orthop Sports Phys Ther. 1996;24(2):57-65. doi:10.2519/jospt.1996.24.2.57

22. Ludewig PM, Reynolds JF. The Association of Scapular Kinematics and Glenohumeral Joint Pathologies. J Orthop Sports Phys Ther. 2009;39(2):90-104. http://www.jospt.org/doi/10.2519/jospt.2009.2808doi:10.2519/jospt.2009.2808

23. McClure PW, Michener LA, Karduna AR. Shoulder Function and 3-Dimensional Scapular Kinematics in People With and Without Shoulder Impingement Syndrome. Phys Ther. 2006;1075-1091.

http://web.a.ebscohost.com.ezproxy.staffs.ac.uk/ehost/detail/detail?vid=14\&sid= b956e79f-419b-4bc6-8675-d4b464463536\%40sessionmgr4008\&hid=4101\&bdat $\mathrm{a}=J n N p d G U 9 Z W h v c 3 Q t b G 12 Z Q \% 3 D \% 3 D \# A N=21802032 \& d b=s 3 h$

24. McClure PW, Michener LA, Sennett BJ, Karduna AR. Direct 3-dimensional measurement of scapular kinematics during dynamic movements in vivo. J Shoulder Elbow Surg. 2001;10(3):269-277. doi:10.1067/mse.2001.112954

25. Meyer KE, Saether EE, Soiney EK, Shebeck MS, Paddock KL, Ludewig PM. 
Scapular angles with different trunk postures

Three-Dimensional Scapular Kinematics During the Throwing Motion. J Appl Biomech. 2008;24(1):24-34.

26. Miyashita K, Kobayashi H, Koshida S, Urabe Y. Glenohumeral, Scapular, and Thoracic Angles at Maximum Shoulder External Rotation in Throwing. Am J Sports Med. 2010;38(2):363-368. http://journals.sagepub.com/doi/10.1177/0363546509347542doi:10.1177/036354 6509347542

27. Miyashita K, Urabe Y, Kobayashi H, Yokoe K, Koshida S, Kawamura M, et al. The role of shoulder maximum external rotation during throwing for elbow injury prevention in baseball players. J Sports Sci Med. 2008;7(2):223-228.

28. Myers JB, Pasquale MR, Laudner KG, Sell TC, Bradley JP, Lephart SM. On-the-field resistance-tubing exercises for throwers: An electromyographic analysis. J Athl Train. 2005;40(1):15-22. doi:10.1016/S0162-0908(08)70347-7

29. Nagai K, Tateuchi H, Takashima S, Miyasaka J, Hasegawa S, Arai R, et al. Effects of trunk rotation on scapular kinematics and muscle activity during humeral elevation. J Electromyogr Kinesiol. 2013;23(3):679-687. http://dx.doi.org/10.1016/j.jelekin.2013.01.012doi:10.1016/j.jelekin.2013.01.012 30. Okamoto S, Endo Y, Saito Y, Nakazawa R, Sakamoto M. Three-dimensional 
Scapular angles with different trunk postures

kinematic analysis of glenohumeral , scapular , and thoracic angles at maximum shoulder external rotation associated with baseball shadow pitching : comparison with normal pitching. J Phys Ther Sci. 2018;30(7):938-942. doi: 10.1589/jpts.30.938.

31. Oliver G, Weimar W. Scapula kinematics of youth baseball players. J Hum Kinet. 2015;49(1):47-54. doi:10.1515/hukin-2015-0107

32. Orozco-Levi M, Gea J, Monells J, Aran X, Aguar MC, Broquetas JM. Activity of latissimus dorsi muscle during inspiratory threshold loads. Eur Respir J. 1995;8(3):441-445. doi:10.1183/09031936.95.08030441

33. Pascoal AG, Morais N. Kinematic comparison and description of the 3-dimensional shoulder kinematics of 2 shoulder rotation tests. J Manipulative Physiol Ther. 2015;38(4):288-294. doi:10.1016/j.jmpt.2014.10.017

34. Shaheen AF, Bull AMJ, Alexander CM. Rigid and elastic taping changes scapular kinematics and pain in subjects with shoulder impingement syndrome: an experimental study. J Electromyogr Kinesiol. 2015;25(1):84-92. http://dx.doi.org/10.1016/j.jelekin.2014.07.011doi:10.1016/j.jelekin.2014.07.011

35. Solomito MJ, Garibay EJ, Woods JR, Õunpuu S, Nissen CW. Lateral trunk lean in pitchers affects both ball velocity and upper extremity joint moments. Am J 
Scapular angles with different trunk postures

576

577

578

579

580

581

582

583

584

585

586

587

588

589

590

591

592

593

Sports Med. 2015;43(5):1235-1240. doi:10.1177/0363546515574060

36. Struyf F, Nijs J, Baeyens JP, Mottram S, Meeusen R. Scapular positioning and movement in unimpaired shoulders, shoulder impingement syndrome, and glenohumeral instability. Scand J Med Sci Sports. 2011;21(3):352-358.

doi:10.1111/j.1600-0838.2010.01274.x

37. Werner SL, Fleisig GS, Dillman CJ, Andrews JR. Biomechanics of the Elbow During Baseball Pitching. J Orthop Sports Phys Ther. 1993;17(6):274-278. http://www.jospt.org/doi/10.2519/jospt.1993.17.6.274doi:10.2519/jospt.1993.17. 6.274

38. Wu G, Van Der Helm FCT, Veeger HEJ, Makhsous M, Van Roy P, Anglin C, et al. ISB recommendation on definitions of joint coordinate systems of various joints for the reporting of human joint motion-Part II: shoulder, elbow, wrist and hand. J Biomech. 2005;38(5):981-992. doi:10.1016/j.jbiomech.2004.05.042

39. Yamauchi T, Hasegawa S, Matsumura A, Nakamura M, Ibuki S, Ichihashi N. The effect of trunk rotation during shoulder exercises on the activity of the scapular muscle and scapular kinematics. J Shoulder Elbow Surg. 2015;24(6):955-964. http://dx.doi.org/10.1016/j.jse.2014.10.010doi:10.1016/j.jse.2014.10.010 
Scapular angles with different trunk postures

594

595

596 Table legends

597 Table I. Kinematics data

598 Table II. EMG data

599

600 Figure I. Trunk postures

601 